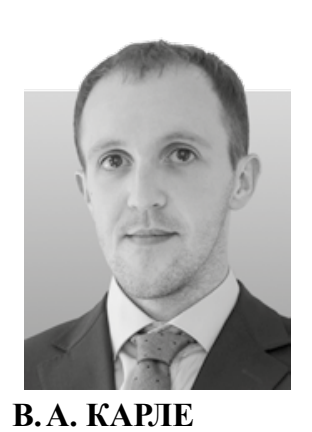
В.А. КАРЛЕ
Заместитель директора
Центра отраслевых исследований и консалтинга Финансового университета при Прауниверситета при ПраФедерации. Область научных интересов: экономика электроэнергетики, ценообразование, инвестиционная деятельность в электроэнергетике, система лирования ест

E-mail: karlevadim@mail.ru

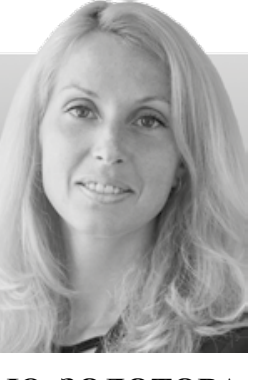

и.Ю. ЗОЛОТОВ Директор Центра отраслевых исследоФинансового универстета при Правительстве Российской Федерашии Область научных интересов: система государственного регулирования естественньх монополий, ценообразование, модели прогнозирования цен в электроэнергетике и инфраструктурных отраслях

E-mail: izolotova1977@
ОСНОВНЫЕ ПРИНЦИПЫ ТЦА ИП И ОСОБЕННОСТИ ЕГО ПРОВЕАЕНИЯ

Для осуществления мероприятий, предусмотренных ТЦА, утверждены Методические рекомендации по прове-
дению ТЦА ИП (проектов ИП) и отчетов об их реализации [Распоряжение, 2016].

При этом процедура и содержание значительно отличаются от предусмотренных в рамках публичного ТЦА крупных инвестиционных проектов с государственным участием. Публичный технологический и ценовой аудит отдельных инвестиционных проектов электросетевые организаци проводили и до внесения в 2015 году изменений в «Правила утверждения инвестиционных программ субъектов электроэнергетики». Тогда же в рамках процедуры согласования и утверждения ИЙ электросетевые орғанизации начал представлять первые заключения по результатам ТЦА в со-

( с государственным участием, в рамках ТЦА ИП (проекто ИП) полагается проводить анализ и составля (проектов для всех титулов, включенных в ИП (проект ИП), с целью:

- проверить соответствие ИП (проекта ИП) требованиям законодательства Российской Федерации, предьявляемым к инвестиционной деятельности сетевых организаций;

- оценить необходимость и достаточность инвестиционных проектов, планируемых к реализации в рамках значений количественных показателей ИП;

- проверить выполнение требований законодательств Российской Федерации к ценовым и (или) стоимост-

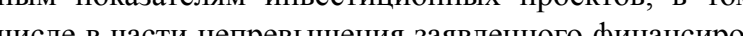
вания относительно объема финансирования, определенного в соответствии с укрупненньми нормативами цены (УНЦ) типовых технологических решений қапи- гального строительства объектов электроэнергетики;

подготовить по результатам проведенных провероз и выполненных оценок предложения по внесению изменений в ИП (проект ИП).

Заключение составляется в целом для ИП (проекта ИП) по совокупности инвестиционных проектов, но проведени проверки по ряду пунктов методических рекомендаций требует подробного рассмотрения параметров отдельных титулов ИП. Так, например, в рамках ТЦА ИП (проекта ИП) обоснованность финансовых потребностей на реализацию инвестиционных проектов проверяется по огдельным объекraм. В рамках дарой порерки аудитору необходимо оценит состав материалов,

- для инвестиционных проектов с утвержденной проектной документашией: свонка затрат сводный сметныи рой докут, пояснительная записка к сметной документации, разработаннье в составе утвержденной проект- ной документации, копия решения об утверждении проектной документации;

для инвестиционных проектов, находящихся в стадии проектирования: сметный расчет стоимости реализации инвестиционного проекта и копии документов формании для полготовки сметного расчета (за иснлючением укрупненных сметных нормативов $)^{1}$.

Подробное рассмотрение параметров отдельных титулов ИП в рамках ТЦА ИП (проекта ИП) требует времени, а оно ограничено исходя из процедуры согласования ИП электросетевых организаций.

Согласно Методическим рекомендациям [Распоряжение, 2016], сроки подготовки и передачи экспертной организацией сетевой организации заключения по результатам проведения ТЦА ИП (проектов ИПП) должны составлять не менее 30 календарных дней, при этом обеспечивать возможность опубликовать информацию в открытом доступе на соответствующих ингернег-ресурсах о проведенном ТЦА в установлеС учетом отавление, 2004, Постановление 2011]. евой организамией, проектов ИП, исходя из потученнех замечаний и превлоиений, в рамках промежугонних этапов процелуры согласования, у аудитора остается не так много времени на полготовку заключения аудитора - примерно 15-20 дней на каждый этап.

У различных электросетевых компаний количество титулов ИП (проекта ИП) варьируется (число титулов может достигать 15000 и более), времени на анализ отдельных инвестиционных проектов остается еще меньше, иногда тщательная проверка материалов оказывается невозможной. Ограничение времени вынуждает аудитора искать оптимальные способы учесть требования Методических рекомендаций, собрать данные и провести ТЦА. При проведении технологического и ценового аудита ИП (проекта ИП) аудитор должен использовать достоверные данные и применять на-

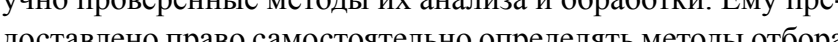
. зультатов ТЦА.

B жет сокращать количество рассматриваемых форм ИП, он оскумендолжен проверять весь комплект обосновывающих документов ТЦА, и не может делать выводы на основе только его части.

Применяя методы отбора данных, подлежащих проверке для формирования заключения по ИП (проекту ИП) в целом, в рамках ТЦА допустимо менять лишь анализируемый перечень титулов ИП. Для проверки аудитор должен отобрать ИГ таким образом, чтобы они представляли репрезентативную выборку и выводы на основе ее анализа могли быть обоснованно распространены на весь ИП (проект ИП). Для проветел таге ( тулов ИП как метод отбора данних, полпежаних проверке для достижения целевых результатов ТЦА. задача найти оптимальный метод отбора и анализа данных, с одной стороны, повышающий эффективность проведения ТЦА, а с другой - решающий ключевую задачу комплексной проверки ИП (проекта ИП) и подготовки обоснованного заключении со стороны экспертной организации. 
МЕТОАИКА ФОРМИРОВАНИЯ

РЕПРЕЗЕНТАТИВНОЙ ВЫБОРКИ

Репрезентативная выборка - это выборка конечного объема, в которой все основные признаки генеральной совокупности представлены приблизительно в той же пропорции в этой генеральной совокупности [4]. Репрезентативность в этой генеральной совокупности [4]. Репрезентативность лиза выборки на всю генеральную совокупность

Прежде всего, необходимо определить, какое количество объектов в выборке является необходимым и достаточным С этой целью можно построить, например, доверительный интервал математического ожидания генеральной совокупности. Данный подход позволяет с заданной точностью определить границы, в пределах которых выборочное среднее отличается от истинного среднего (математического
ожидания) генеральной совокупности. Метод применим ожидания) генеральной совокупности. Метод применим при наличии нормального распределения генеральной содения объема репрезентативной выборки следует учесть допустимую величину ошибин вибороннго нсследовани и задать доверительный уровень. Также важным показатеотентонение от среднего значения лем является стандар

Для вычисления объема репрезентативной выборки используется формула нахождения доверительного интервала для математического ожидания (среднего значения случай-
ной величины) [Боровков А. А., 1984; Левин Д. М., 2004]:

$$
\bar{x}-Z * \frac{\sigma}{\sqrt{n}}<\mu<\bar{x}+Z * \frac{\sigma}{\sqrt{n}},
$$

где $\bar{x}$ - выборочное среднее; $Z$ - квантиль стандартноо нормального распределения; $\sigma$ - стандартное отклонени генеральной совокупности; $n$ - объем выборки; $\mu$ - истинно (ости). Слагаемое и вычитаемое относительно $\bar{x}$ солуг ности). Слагамме и вычитаемое относительно $\bar{x}$ соответснучение определяет меру неточности оценки, возникаюше вследствие ошибки выборочного исследования $e$ :

$$
e=Z * \frac{\sigma}{\sqrt{n}} .
$$

Зная составляющие данного равенства, можно определить объем выборки $n$ :

$$
n=\left(\frac{Z * \sigma}{e}\right)^{2} .
$$

С учетом исходной неравномерности распределения случайной величины в рамках генеральной совокупности применяются районирование и кластеризация. Данные подходы териям. В дальнейшем с учетом необходимого и достаточного объема репрезентативной выборки по совокупности всех кластеров посредством случайного выбора (например, в ис- пользованием специальных алгоритмов типа Random) отбираются конкретные элементы внутри однородных групп, представляющие репрезентативную выборку генеральной совокупности (района/кластера). В выделяемых кластерах анализируемый признак может быть распределен не по нор-
мальному закону распределения, что потребует использования других методов формирования репрезентативной выборки.

\section{ПРИМЕР ФОРМИРОВАНИЯ}

РЕПРЕЗЕНТАТИВНОЙ ВЫБОРКИ

ААЯ ПРОВЕАЕНИЯ ТЦА

Рассмотрим применение методологии с определением необходимых параметров для формирования репрезентативной выборки на примере проекта ИП ПАО «МОЭСК», В качестве исходных данных примем информацию о проек[б.г.]].

Проект ИП ПАО «МОЭСК» содержит 13532 отдельных титула, их полная стоимость строительства составляет 442, млрд руб. в прогнозных ценах соответствующих лет. Инвестиционные проекты ПАО «МОЭСК» реализуются на территории двух субъектов Российской Федерации (Москва и Московская область), соответственно, ИП сетевой орган Для формирования репрезентативной выборки источниДля формирования репу о сат вложений по инвестиционным проектам» [Приказ, 2016 прилож. 2]. В ней привелены порная структура и перечен прилож. 2]. В ней приведены полная структура и перечень ризующих инвестиционные проекть

Ключевым параметром отбора инвестиционных проекгов для формирования репрезентативной выборки, который в данном случае рассматривается в качестве случайной величины, является оценка полной стоимости инвест проектов в прогнозных ценах соответствующих лет (графа 18 формы 2) (далее - полная стоимость инвестиционного

Для формирования однородных по случайной величине групп инвестиционных проектов из общей структуры проекта ИП ПАО «МОЭСК» в рамках процесса районирования

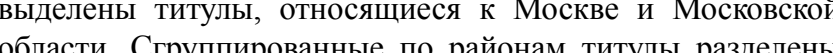
на кластерн в соответствии с группами инесстишионше проектов по направлениям их реализачии.

Перечень кластеров состоит из семи групп инвестиционных проектов, соответствуюших направтениям их реализа-

устройств мощностью свыше 150 кВт;

реконсрукция существующих объектов электросете- лас осудеспвения технологического присоединения; - реконструкция, модернизация, техническое перевооружение трансформаторных и иных подстанций, рас-

- реконструкшия линий электропередачи;
- прочее новое строительство объектов электросетевого хозяйства;

- группа «прочие инвестиционные проекты»;

- проекты, не вошедшие в перечисленные группы. Ввиду наличия относящ вися к Московской области тигехническому перевооружению линий эгентрогерепачи, для области выделен соответствуюший отдельный кладля о

С С учетом сильного разброса значений полной стоимости инвестиционных проектов распределения по кластерам недостаточно для формирования репрезентативной выборки. Внутри перечисленных кластеров инвестиционных проектов сформированы дополнительные кластеры (подгруппы). При проведении дополнительной кластеризации учитывлось то, какое распределение случайной величины формируется в подгруппах

Рассмотрим кластер инвестиционных проектов «Реконструкция существующих объектов электросетевого хозяйства для усил сй электрической сети в целях осуществлек Москве.

Рассматриваемый кластер включает 683 инвестиционных проекта с полной стоимостью от 0,01 млн руб. до 4295,47 млн руб. Исходя из диапазона полной стоимости инвестици(см. таблицу).

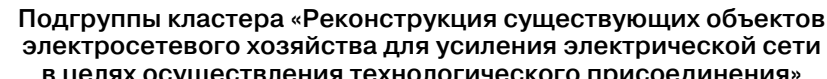

\section{Парамет}

Подгруппа

Пубб.

менее 6 от 6 до 17 более 17

Количество объее
в подгруппе, ед.

Среднее значен

случайной величины
по подгруппе, млн руб.

$$
370
$$

$1,58 \quad 10,8$

480,99

Распределение случайной величины в подгруппах 1 и близко к нормальному (для определения закона распредеблизко к нормаредась графики эмпирической фусед плотности случайной величины). Стандартное отклонение случайной величины от среднего значения для полгруппи случайной величины от среднего значения для подгруппь 1,49 млн руб., для подгруппы $2-2,48$ млн руб.

Для подтверждения того, что в выделенной подгруппе случайная величина распределена по нормальному закону, помимо анализа графика эмпирической функции плотност рекомендуется проверка статистических гипотез, наприме

В рамках статистических исследований при формировании доверительного интервала случайной величины используют доверительные уровни от 90 до 99\%. Мы рекомендуе доверительнй уровень не мене $95 \%$, Н $Z=1,96$
На практике ошибка выборочного исследования генеральной совокупности определяется экспертным путем С учетом значений математического ожидания случайно величины в подгруппах, а также стандартных отклонени ема репрезентативной выборки рекомендуется использовать ошиббу выборочного исследования не более 1 млн руб. лля групп с относительно невысокой полной стоимостьо инвестиционного проекта. Чем меньше значение математического ожидания, тем меньше должно быть значение ошибки выборочного исследования. Для подгруппы 1 мы примем приемлемую ошибку равной 0,6 млн руб.,, для полгруппь $2-1,0$ млн руб. Объем репрезентативной выборки для подгруппы 1 составил 24 инвестиционных проекта, для подгуппы 2 - 22 инвестиционных проекта (рис. 1).

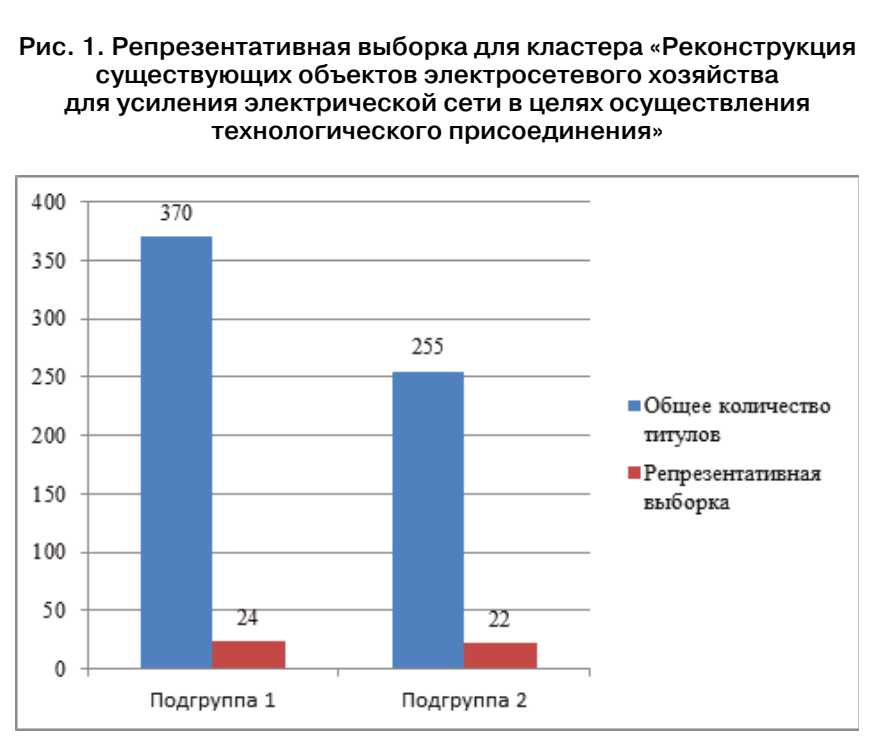

Распределение случайной величины в подгруппах 1 и 2 близко к нормальному, поэтому объем репрезентативной вьборки определен по формуле (3). Для таких подгрупп в рамках кластера конкретные инвестиционные проекты, попадающи в репрезентативную выборку и подлежащие проверке в рамках ТЦА, определяются посредством случайного отбора.

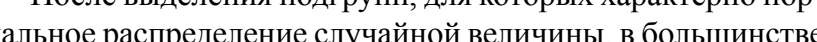
случеев остается значительный массив титупов ИП, для которых не характерно такое распрелеление. Для постелних предлагается выделять дополнительные полгруппы с распределением случайной величины, близким к равномерному и подгруппы с инвестишионными проектами, для которьх характерна высокая полная стоимость инвестиционных проектов (значительно отличающаяся величина относительно большей части (более $90 \%$ ) титулов ИП).

В рамках рассмагриваемого ками вра выделена подгруппа 3 с инвестиционными проектами, для которых характерн высокая полная стоимость инвестиционных проектов - более 17 млн руб. Отбор титулов в рамках таких подгрупп рекомендуется осуществлять экспертным путем. Для опреде ления количества титулов, попадающих в репрезентативную векку, рекомендуеся ориенировагся на процентну 3. Тано з 
методологии. При определении конкретных титулов ИП, подлежащих проверке в рамках ТЦА, из подгрупп, для которых характерна полная стоимость инвестиционных проектов, рекомендуегся отбирать ийуль с наибольшей полно иостаточной стоимоспи

Для подгрупп, где распределение случайной величинь близко к равномерному, определение количества инвестинионных проектов, попадающих в репрезентативную выборку, предлагается осуществлять эмпирически (5-10 инвестишионных проектов). Для таких подгрупп отбор конкретных инвестиционных проектов осуществляется путем случайного отбора. При данном распределении случайный отбор позволит сформировать выборочное среднее близко к истинному значению среднего всей подгруппы.

ентативной выборк для проекта ИП ПАО «МОЭСК» отображены на рис. 2.

\section{Pис. 2. Сокращение объема выборки титулов
для проверки в рамках ТЦА на примере проекта
инвестиционной программы ПАО «МОЭСК»}

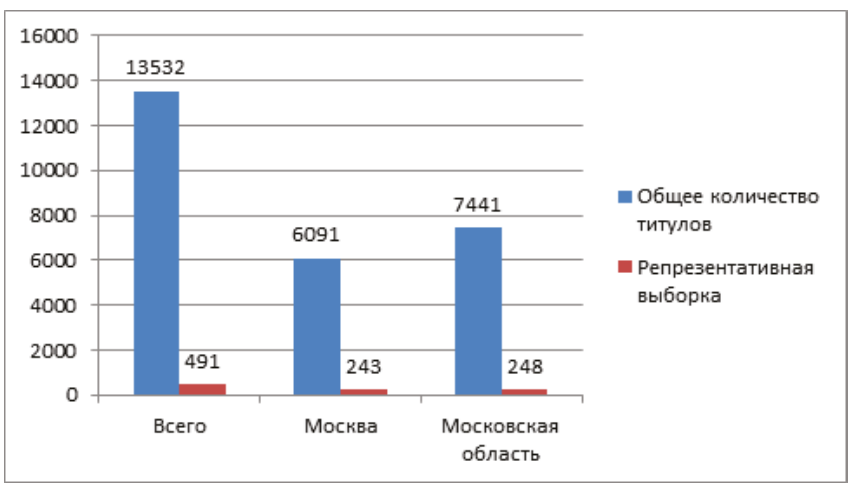

В результате применения предлагаемого подхода к отбору инвестиционных проектов для выполнения ТЦА ИГ (проектов ИП) объем анализируемых инвестиционных проектов сократился более чем в 20 раз, что позволяет повысить эффективность и качество проведения процедур ТЦА. Выобоснованы для генеральной совогупности (иий выборки ной программы в целом).

\section{BЫBOAЫ}

На примере проекта ИП ПАО «МОЭСК» показано, что предлагаемая методология позволяет существенно сократить временные затраты на ТЦА ИП (проектов ИП) электросетевых организаций, повысить результативность, эффективность проверки, оптимизировав проверку в целом. Такой подход не нарушает положений методических рекомендаций по проведению ТЦА. Для соблюдения репрезентативности при использовании предлагаемой методологии ризации инвестиционных проектов при выделении однородных по своим признакам групп (кластеров) инвестиционных проектов, соответствующих распределению случайной величины.

\section{АИТЕРАТУРА}

Боровков A.A. (1984) Математическая статистика. Оценка параметров, проверка гипотез. М.: Наука. $472 \mathrm{c}$. тистика для менеджеров. М.: Вильямс. $1312 \mathrm{c}$.

Лемешко Б.Ю. (2014) Критерии проверки отклонения распределения от нормального закона: Руководство по применению. Новосибирск: Новосиб. гос. техн. ун-т. $160 \mathrm{c}$.

4. Мангейм Джк. Б., Рич Р.К. (1997) Политология. Методы исследования/Пер. с англ.; предисл. А. К. Соколова. М. Весь мир. $544 \mathrm{c}$.

5. ПАО «МОЭСК» ([б...])// Министерство энергетики Российской Федер

6. Постановление Правительства РФ от 16.02.2015 № 132 (рен. от 17.02 2017) ний в некоторые акты Правите геств Российской Федерашии по вопросам утверждения инвестиционных программ суббектов электроэнергетия и контроля за их реализацией» // КонсультантПлюс. URL: http://www consultant.ru/document/cons doc LAW_175479/.

7. Постановление Правительства РФ от 21.01.2004 № 24 (ред. от 07.06.2017) «Об утверждении стандартов раскрытия информации субъектами оптового и розничных рынков электрической энергии» (с изм. и доп., вступ. в силу с 29.09.2017) // КонсультантПлюс. URL: htp./MWWW Co LAW_46197/.

8. Постановление Правительства РФ от 28.11.2011 № 977 (ред. от 30.06.2018) «О федеральной государсленнй информационной сислеме қЕдиная система обеспечивающей информашионно-технологическое взаимодействие ин орманионных систем, используемых для предоставления государственных и муниципальных услуг в электронной форме»» // КонсультантПлюс. URL: http://www.consultant.ru/document/cons doc LAW_122455/.

9. Приказ Минэнерго России № 380 от 05.05.2016 «Об утверждении форм раскрытия сетевой организацией информации об инвестиционной программе (о проекте инвестиционной программы и (или) проекте изменений, вносимых в инвестиционную программу) и обосновывающих ее материалах, указанной в абзацах втором - четвертом, шестом, восьмом и десятом формашии суб ентами огтового и розничнех ренгов электрической энергии, утвержленних постановлением Правительства Российской Федерачин от 21 января 2004 г. № 24 , правил заполнения указанных форм и требований к форматам раскрытия сетевой организацией электронных документов, содержаших информацию об инвестиционной программе (о проекте инвестиционной программы и (или) проекте изменений, вносимых в инвестиционную программу) и обосновываюших ее материатах» // Консультантww.consultant.ru/document/cons doc_LAW_199581/.

10. Распоряжение Правительства РФ от 23.09.2016 № 2002-p «Об утверждении методических рекомендаций по проведению технологического и ценового аудита инвестиционных программ (проектов инвестиционных программ) сетевых организаций, отнесенных к числу субъектов электроэнергетики, инвестиционные программы которых утверждаются Министерством энергетики Российской Федерации и (или) органами исполнительной власти субьектов Российской Федериоле и отчетов об их реализашии» // КонсультантПлюс. URL: http://wwwconsultant ru/document/cons_doc_ LAW_205249/.

11. Четыркин Е. М., Калихман И. Л. (1982) Вероятность и статистика. М.: Финансы и статистика. 319 с. 